\title{
De novo expression of CD44 variants in sporadic and hereditary gastric cancer
}

\author{
Cristiana Branco da Cunha ${ }^{1,2}$, Carla Oliveira ${ }^{1,3}$, Xiaogang Wen ${ }^{1,4}$, Bárbara Gomes ${ }^{1,4}$, Sónia Sousa , \\ Gianpaolo Suriano ${ }^{1,3}$, Maritie Grellier ${ }^{1}$, David G Huntsman ${ }^{5,6}$, Fátima Carneiro ${ }^{1,3,4}$, Pedro L Granja ${ }^{2,7}$ \\ and Raquel Seruca ${ }^{1,3}$
}

CD44 is the major ubiquitously expressed cell surface receptor for hyaluronate. The CD44 gene encodes several protein isoforms due to extensive alternative splicing and post-translational modifications. Some of these CD44 variable isoforms have been foreseen as key players in malignant transformation and their expression is highly restricted and highly specific, unlike the canonical CD44 standard isoform. In this study, we aimed at dissecting the mRNA splicing pattern of CD44 in normal stomach and gastric cancer (GC) cell lines $(n=9)$ using cloning and quantitative mRNA amplification assays. Moreover, we assessed the RNA levels and protein expression pattern of relevant splicing forms in distinct premalignant and malignant gastric lesions (sporadic $(n=43)$ and hereditary $(n=3)$ forms) using real-time RT-PCR and immunohistochemistry. We also explored the association of CD44 and E-cadherin expression by immunohistochemistry, as E-cadherin has a pivotal functional role in GC. We established the pattern of CD44 variant forms in normal stomach and gastric malignancy. We observed that although exon v6-containing isoforms were rarely expressed in normal gastric mucosa, they became increasingly expressed both in gastric premalignant (hyperplastic polyps, complete and incomplete intestinal metaplasia, low- and high-grade dysplasia) and malignant lesions (cell lines derived from GCs, primary sporadic GCs and hereditary diffuse GCs (HDGCs)). Moreover, we verified that whenever E-cadherin expression was absent, exon v6-containing CD44 isoforms were overexpressed. The lack of expression of CD44 isoforms containing exon v6 in the surface and foveolar epithelia of normal stomach and, its de novo expression in premalignant, as well as in sporadic and hereditary malignant lesions of the stomach, pinpoint CD44 v6-containg isoforms as potential biomarkers for early transformation of the gastric mucosa. Further, our results raise the hypothesis of using CD44v6 as a marker of early invasive intramucosal carcinoma in HDGC CDH1 mutation carriers that lack $\mathrm{CDH} 1$ expression in their tumors.

Laboratory Investigation (2010) 90, 1604-1614; doi:10.1038/labinvest.2010.155; published online 20 September 2010

KEYWORDS: CD44; CD44v6; E-cadherin; HDGC; splicing; sporadic GC

CD44 is the major cell surface receptor for hyaluronate, ${ }^{1}$ and has been implicated in several cellular mechanisms, from lymphocyte homing to tumor dissemination. The CD44 human gene (NG_008937) structure was initially annotated with at least 19 exons spanning $50 \mathrm{~kb}$ of genomic DNA. ${ }^{2}$ This gene is a target of complex splicing, with 10 of the 19 exons (the first five and the last five exons) being kept in a ubiquitously expressed (both in epithelial and nonepithelial tissues) splicing form named CD44 standard (CD44s). The remaining nine internal variable in-frame exons (from exon 6 to 14) can be individually spliced in or -out in the CD44 mRNA transcripts, originating over 100 different combinations. ${ }^{3}$

The translation of the 10 constant exons of the CD44s transcript produces a transmembrane protein that is exposed at the surface of most mammalian cells. The CD44s extracellular domain is encoded by the initial seven exons. Different combinations between the 10 constant exons and

${ }^{1}$ IPATIMUP, Institute of Molecular Pathology and Immunology of the University of Porto, Porto, Portugal; ${ }^{2}$ INEB, Instituto de Engenharia Biomédica, Universidade do Porto, Porto, Portugal; ${ }^{3}$ Medical Faculty of the University of Porto, Porto, Portugal; ${ }^{4}$ Department of Pathology, Hospital S. João, Porto, Portugal; ${ }^{5}$ British Columbia Cancer Agency, Vancouver, Canada; ${ }^{6}$ Department of Pathology and Laboratory Medicine, University of British Columbia, Vancouver, Canada and ${ }^{7}$ Engineering Faculty of the University of Porto, Porto, Portugal

Correspondence: Dr R Seruca, MD, PhD, IPATIMUP, Institute of Molecular Pathology and Immunology of the University of Porto, Rua Dr. Roberto Frias s/n, $4200-465$ Porto, Portugal.

E-mail: rseruca@ipatimup.pt

Received 28 August 2009; revised 23 June 2010; accepted 24 June 2010 
the 9 variable exons of the $C D 44$ gene result in changeable structures, affinities and functions. The expression of CD44 variable isoforms is known to be restricted mainly to highproliferating cells in epithelial normal tissues. ${ }^{4}$

CD44 has been extensively studied in several cancer types, although its role in carcinogenesis remains controversial. In some types of cancer it can act as a tumor suppressor, as in prostate carcinomas or neuroblastomas, ${ }^{5,6}$ whereas in others it can act as a tumor promoter, as in breast cancer, ${ }^{7-9}$ colorectal cancer ${ }^{10,11}$ or non-Hodgkin's lymphomas. ${ }^{12}$

Recently, in gastric carcinoma (GC), it was shown that the $\mathrm{CD} 44(+)$ subpopulations of gastric malignant cell lines give rise to xenograft tumors in SCID mice. ${ }^{13}$ Heider et al ${ }^{14}$ described the expression of CD44 variant isoforms in sporadic gastric tumors and specifically CD44v6 was indicated as a potential marker to distinguish intestinal- and diffuse-type stomach carcinomas. Since then, most reports described the expression of CD44 variants and their association with the distinct histotypes of this neoplasia in comparison with normal mucosa, without further considerations. ${ }^{15-20}$

In this study we aimed to dissect the splicing pattern of CD44 in gastric neoplasm in comparison to normal stomach. The expression of relevant splicing forms was further quantified and compared with that of normal gastric mucosa, in terms of RNA and protein, in distinct lesions and stages of GC development: GC cell lines, primary premalignant lesions and malignant gastric lesions (sporadic and hereditary forms). Furthermore, we explored the association of CD44 and E-cadherin expression, as E-cadherin has a pivotal functional role in GC. ${ }^{21-31}$

In hereditary diffuse GC (HDGC), neoplastic cells show absent/aberrant E-cadherin expression and invade the gastric wall either isolated or in poorly cohesive clusters. HDGC early lesions remain undetected with currently used diagnostic tools. ${ }^{32}$ This limitation in cancer detection clinically supports prophylactic gastrectomy as the only reliable medical approach to prevent disease progress in $\mathrm{CDH1}$ mutation carriers belonging to HDGC families. ${ }^{27}$

We identified overexpression of CD44 molecules in GC (GC cell lines, primary sporadic gastric carcinoma and HDGC) and its premalignant lesions (intestinal metaplasia and dysplasia). In hereditary forms of GC (HDGC), our findings may disclose a putative tool for screening and early diagnosis.

\section{MATERIALS AND METHODS \\ Gastric Malignant Cell Lines}

We studied nine GC cell lines: AGS, GP202, IPA220, KATOIII, MKN28, MKN45, NCI-N87, SNU-1 and SNU-638. Both SNU cell lines were maintained in Dulbecco's Modified Eagle's Medium, supplemented with $10 \%$ fetal bovine serum and $100 \mu \mathrm{g} / \mathrm{ml}$ of penicillin/streptomycin. The other seven GC cell lines were maintained in Roswell Park Memorial Institute Medium (RPMI), equally supplemented. All cells were divided using trypsin-EDTA, and cultured at $37^{\circ} \mathrm{C}$ in a humidified incubator with a $95 \%$ air and $5 \% \mathrm{CO}_{2}$ atmosphere. With the exception of KATO-III and SNU-1, all GC cell lines grow as adherent colonies. Absence of mycoplasma contamination was periodically screened by PCR.

\section{Tissue Samples}

Formalin-fixed paraffin-embedded (FFPE) sections were obtained from normal gastric mucosa, premalignant and malignant lesions of the stomach, encompassing the following: normal gastric mucosa $(n=11)$; hyperplastic polyps $(n=8)$; intestinal metaplasia $(n=9)$; adenomatous dysplasia $(n=11)$; and primary sporadic GC $(n=43)$. This material was retrieved from the Department of Pathology of Hospital S. João, under the approval of the Hospital Ethics Committee. Our study was conducted with the informed consent of patients, and in strict accordance with the ethical guidelines of the Institutional Medical Ethics Committee. Diffuse GCs $(n=3)$ belonging to three HDGC families carrying three different germline $\mathrm{CDH} 1$ mutations were provided by the British Columbia Cancer Agency (Vancouver, Canada), with ethical approval and informed consent.

Consecutive 3- $\mu \mathrm{m}$ sections were stained with hematoxylin and eosin and classified by two experienced gastrointestinal pathologists (FC and XW). GCs were classified according to Lauren classification: ${ }^{33}$ intestinal $(n=14)$, diffuse $(n=13)$ and mixed $(n=16)$ types. Intestinal metaplasia was classified as complete and incomplete. ${ }^{34}$

\section{Cloning and Sequencing of CD44 Transcripts}

In order to explore the CD44 alternative splicing pattern in gastric neoplasia, total RNA was isolated from nine GC cell lines when $100 \%$ confluence was reached, using the Tripure Isolation Reagent (Roche Applied Science). Reverse transcription was performed using SuperScript II Reverse Transcriptase KIT (Invitrogen) with $1 \mu \mathrm{g}$ of RNA in a $20-\mu \mathrm{l}$ volume reaction.

CD44 transcripts encoding variable exons were amplified, from GC cell line cDNA and commercially available RNA from normal stomach (Ambion, Applied Biosystems), using a set of primers designed for the CD44 gene constant regions (CD44ex.5F: 5'-CATCCCAGACGAAGACAGTCC-3' and CD44ex.17/18R: $5^{\prime}$-TGATCAGCCATTCTGGAATTTG- $3^{\prime}$ ). RT-PCR products were cloned into a pCR2.1 vector using TOPO PCR Cloning Kit (Invitrogen), and Colony PCR was performed using the universal M13 primers. Colony PCR products were electrophoresed in $2 \%$ agarose gels to distinguish CD44 transcripts with different sizes. To determine the variable exons encoded in each one of these CD44 transcripts, PCR products were retrieved from the agarose gel using the GFX PCR DNA and Gel Band Purification Kit (GE Healthcare), and sequenced using the BigDye Terminator v3.1 Cycle Sequencing Kit (Applied Biosystems). 


\section{RNA Isolation and CDNA Synthesis}

To determine the expression levels of v6/v7-encoding CD44 transcripts in primary sporadic GC, RNA was isolated from $10 \mu \mathrm{m}$ FFPE sections using the RecoverAll Total Nucleic Acid Isolation Kit (Ambion, Applied Biosystems). RNA concentrations were measured using the Nanodrop Spectrophotometer ND-1000 (Thermo Scientific). Commercially available RNA from normal stomach (Ambion, Applied Biosystems) was used as comparison.

Reverse transcription was performed using SuperScript II Reverse Transcriptase KIT (Invitrogen) with the maximum amount of RNA from primary sporadic GC in a 10- $\mu \mathrm{l}$ volume reaction.

\section{Quantification of CD44 v6/v7-Encoding mRNA Transcripts}

Ten cases of primary sporadic GC displaying over $75 \%$ of CD44v6 $(+)$ malignant cells were selected for quantification of the expression levels of v6/v7-encoding CD44 transcripts. All carcinomas and adjacent non-neoplastic tissues were manually macrodissected from $10 \mu \mathrm{m}$ FFPE sections.

To determine the expression levels of v6/v7-encoding CD44 mRNA transcripts, both in GC cell lines $(n=9)$ and selected primary sporadic GC $(n=10)$, we performed quantitative target amplification, using cDNA as template, with TaqMan Gene Expression Assay number Hs01075854_m1, and Human GAPDH Endogenous Control, (both from Applied Biosystems), according to the manufacturer's protocol. Commercially available RNA from normal stomach (Ambion, Applied Biosystems) was used as comparison.

\section{Fluorescent Immunocytochemistry}

Cells were seeded on glass coverslips and fixed in 100\% ice-cold methanol. Binding of the primary antibody to unspecific proteins was avoided by incubation with antibody dilution solution (5\% BSA in PBS) for $1 \mathrm{~h}$ at room temperature. Immunostaining with the primary antibody monoclonal Mouse anti-Human CD44v6 antibody, clone MA54 (Invitrogen) was performed overnight at $4^{\circ} \mathrm{C}$, diluted in the ratio of 1:100 in antibody dilution solution. Binding was detected by incubating with Goat anti-Mouse Alexa Fluor 488 (Invitrogen) for $1 \mathrm{~h}$ at room temperature, diluted in the ratio of 1:500 in antibody dilution solution. The nucleus was stained using Vectashield Mounting Medium with DAPI (Vector Laboratories). Figures were taken under a fluorescence microscope from Zeiss using a $\times 1000$ magnification.

\section{Immunohistochemistry}

CD44v6 and E-cadherin expression levels were analyzed by immunohistochemistry in sequential sections, the former in the whole series and the latter only in GCs (sporadic and hereditary). The primary antibodies used were monoclonal Mouse anti-Human CD44v6, clone MA54 (Invitrogen); and monoclonal Mouse anti-Human E-cadherin, clone 4A2C7 (Invitrogen). Immunostaining with the primary antibodies was done for $30 \mathrm{~min}$ at room temperature (1:200 dilution), and for $1 \mathrm{~h}$ at room temperature (1:50 dilution). The sections were then incubated with ENVision HRP-labeled Goat antiMouse polymer (Dako) for $30 \mathrm{~min}$ at room temperature. Binding was detected using DAB (Dako), which upon oxidation forms a stable brown end product. The membranous pattern of CD44v6 and E-cadherin immunoreactivity was graded according to the percentage of positive cells. A threshold of $>25 \%$ cells displaying immunoreactivity for v6-containing CD44 isoforms was established for the scoring of CD44v6 overexpression. Positive immunoreactivity in the cytoplasm for any of the antibodies was considered aberrant.

\section{Statistical Analysis}

Student's $t$-tests and $\chi$-square were used. A value of $P<0.05$ was considered statistically significant.

\section{RESULTS}

In this study, the alternative splicing of the CD44 gene was explored in GC cell lines and normal gastric mucosa. De novo mRNA and protein expression of CD44 aberrant forms was analyzed throughout GC progression in hyperplastic polyps, intestinal metaplasia, gastric dysplasia and primary GCs (sporadic and hereditary). Furthermore, the analysis of a potential association between CD44 and E-cadherin was explored, as loss/aberrant expression of E-cadherin is a common feature of GC, namely in the diffuse type.

\section{CD44 Alternative Splicing Pattern in Normal Stomach and GC Cell Lines}

A set of primers lying in the constant portions of CD44 mRNA was designed flanking all variable CD44 exons to amplify normal gastric mucosa (commercially available) and nine GC cell lines (AGS, GP202, IPA220, KATO-III, MKN28, MKN45, NCI-N87, SNU1 and SNU638) cDNAs. MKN28 harbored $\mathrm{CD} 44$ promoter hypermethylation and was used as a negative control. ${ }^{35} \mathrm{~A}$ single low-molecular-weight band corresponding to the CD44 standard (CD44s) transcript was detected in normal stomach, whereas most GC cell lines displayed a complex pattern of bands with higher molecular weights (Figure 1).

PCR products from normal stomach and two GC cell lines (NCI-N87 and MKN45) were cloned into a pCR 2.1 vector and sequenced. In normal stomach, the most abundant transcript was the CD44s, followed by very low expression of the so-called epithelial variant (CD44E). In GC cell lines, lowest amounts of CD44s were observed, as CD44E was increasingly abundant. NCI-N87 cell line mimicked the normal stomach pattern of CD44 transcripts, whereas MKN45 expressed a more variable pattern of transcripts, with larger CD44 forms that included exons upstream of v8, namely v6 and/or v7 (Figures 1 and 2).

To quantify and validate the specificity of the v6/v7encoding CD44 transcripts in gastric malignancy, we quantified their expression in RNA from normal stomach tissue 


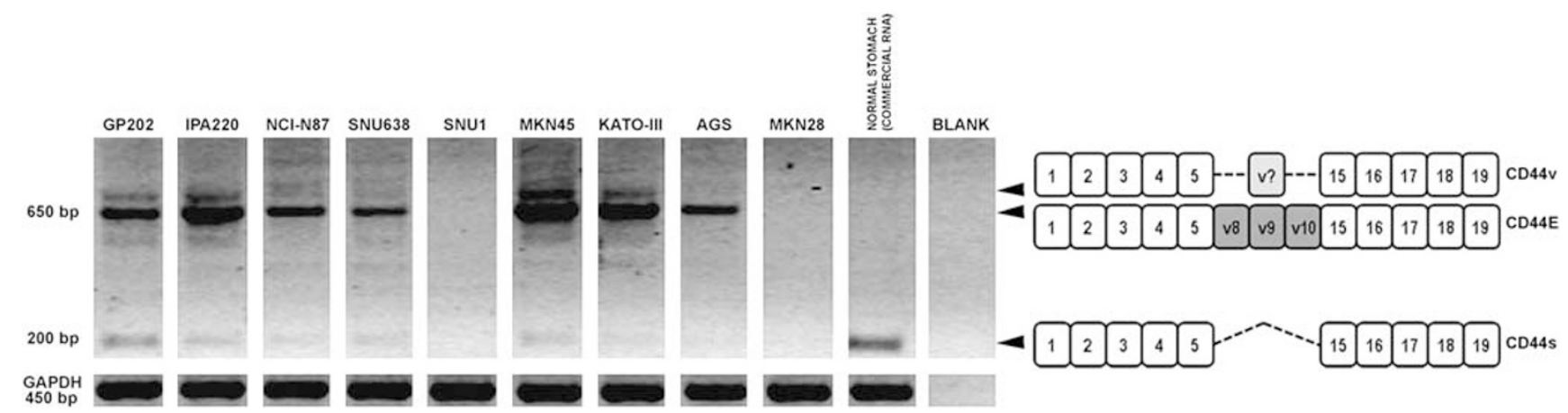

Figure 1 RT-PCR with primers flanking the variable region of CD44 in RNA from nine GC cell lines (GP202, IPA220, NCI-N87, SNU638, SNU1, MKN45, KATO-III, AGS and MKN28) and from normal stomach. Amplification of GAPDH was used as an endogenous control. Blank corresponds to the negative control of the RT-PCR reaction. On the right-hand side, a schematic view of the CD44 transcripts is depicted.

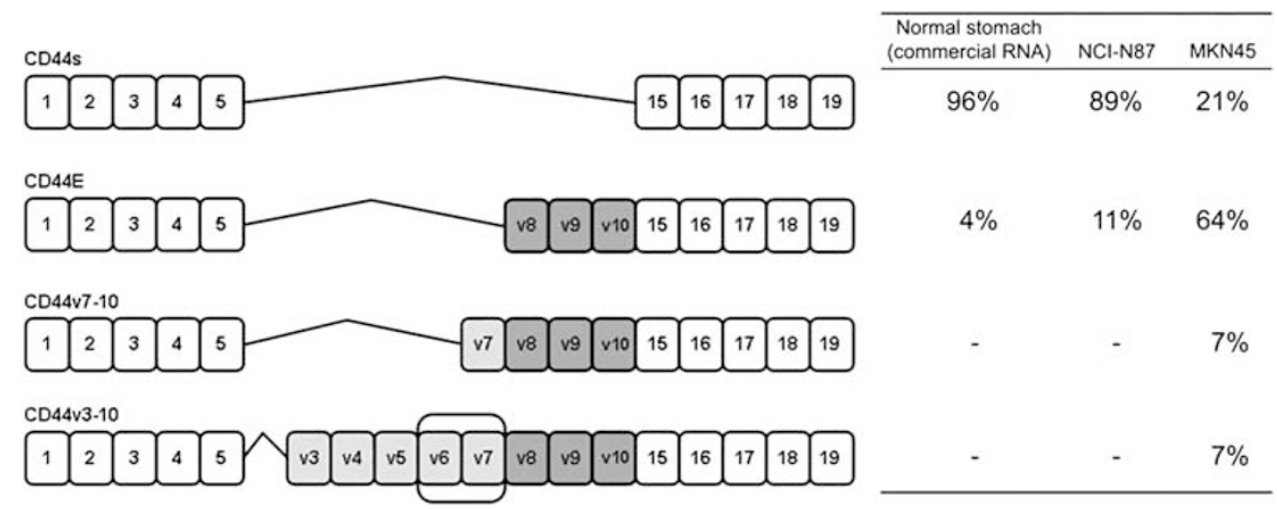

Figure 2 CD44 transcripts cloning in two GC cell lines and normal stomach. The left panel shows schematic representations of different CD44 transcripts; the right panel encloses the relative percentage of colonies that incorporated different CD44 transcripts.

(commercially available) and from nine GC cell lines. This study was performed by real-time PCR using a RT-probe that identifies the splicing junction between exons v6 and v7. Commercial RNA derived from normal stomach showed almost undetectable expression of CD44v6/v7, whereas GC cell lines displayed different levels of expression of this transcript (Figure 3a). SNU-1 and MKN28 completely lacked CD44v6/v7 expression; AGS, KATO-III and SNU638 displayed a CD44v6/v7 expression level similar or lower than that of normal stomach; IPA220 and NCI-N87 displayed moderate levels of expression; and GP202 and MKN45 cell lines showed high levels of CD44v6/v7. In summary, four out of nine (44.4\%) GC cell lines displayed CD44v6/v7 overexpression in comparison with normal stomach.

To verify whether v6/v7-encoding CD44 mRNA transcripts were translated into protein expression, we performed fluorescent immunocytochemistry in all GC cell lines using an antibody that specifically recognizes v6-containing CD44 isoforms. Cell lines displaying low expression levels of v6/v7encoding CD44 mRNA also displayed very low or even absent protein expression. Cell lines showing high v6/v7-encoding CD44 mRNA expression levels showed also high protein expression at the cell membrane (Figure $3 \mathrm{~b}$ ). As observed for v6/v7-encoding CD44 transcripts in normal stomach from cancer free individuals, no CD44v6 protein expression was detected. Therefore, we showed that deregulation of CD44 alternative splicing in gastric neoplasia leads to de novo expression of v6/v7-encoding CD44 transcripts that are translated into aberrant CD44v6 isoforms.

\section{v6-Containing CD44 Isoforms are Expressed in PreMalignant Lesions of the Stomach}

Immunoexpression of CD44v6 was assessed in the whole series of cases. In normal gastric mucosa, CD44v6 expression was very faint, localized in cells of the neck zone of gastric glands and, focally, in deep glands of the antrum (Figure 4a). No expression was observed at the foveolar and surface epithelium of normal gastric mucosa. In hyperplastic polyps, CD44v6 expression was heterogeneous, mainly displayed in elongated/hyperplastic foveolae and, focally, in mucous glands (Figure 4b). In intestinal metaplasia, CD44v6 expression was analyzed separately in complete and incomplete types: in complete intestinal metaplasia, CD44v6 expression was faint and observed in $<25 \%$ of columnar cells 
a

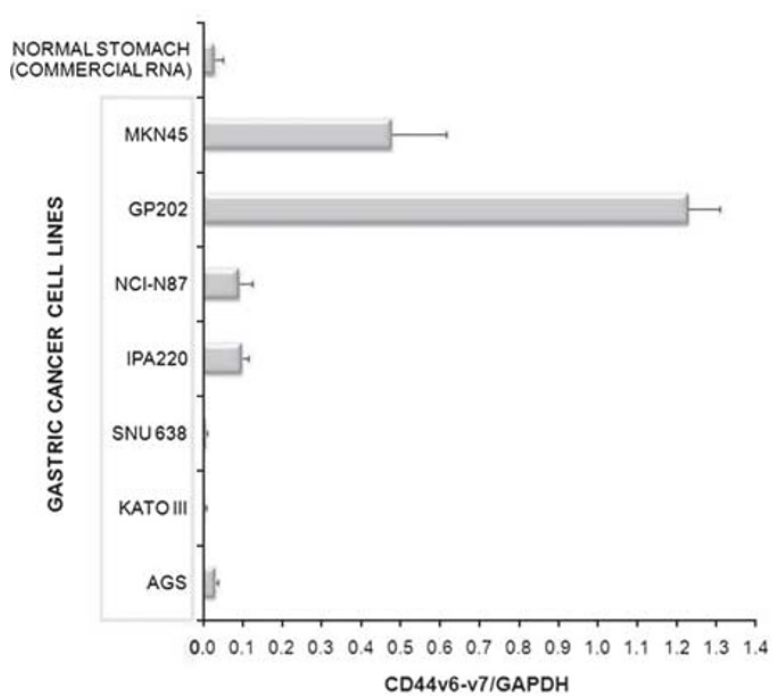

b
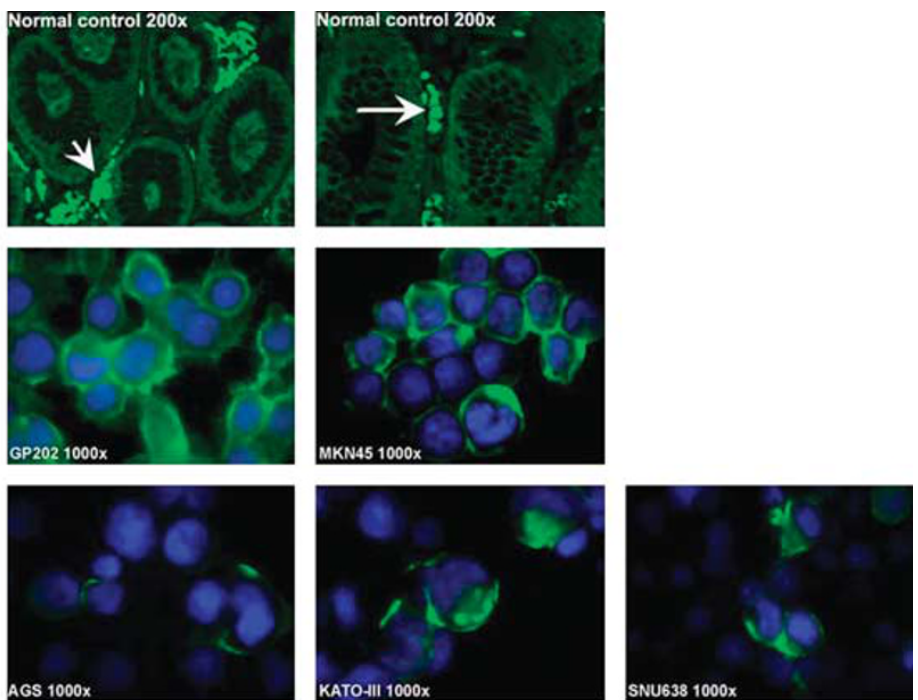

Figure 3 Expression quantification of v6/7-encoding CD44 transcripts and CD44v6 protein isoforms in GC cell lines. (a) Quantitative target amplification of CD44v6/v7 mRNA transcripts performed using TaqMan gene expression assay. Normalization of the values of mRNA transcripts expression was made using human GAPDH endogenous control and s.d. values are displayed. RNA from normal stomach was used as control. Data represent two independent experiments using two independent cell passages, each done in triplicate. (b) Fluorescent immunocytochemistry (green) of CD44v6 in two FFPE GC mucosas from cancer-free individuals and five GC cell lines (GP202, MKN45, AGS, KATO-III, SNU638). DAPI was used to label the nucleus. All images were taken with an amplification of $\times 1000$, and data are representative of at least three independent experiments.

(Figure 4c); in incomplete intestinal metaplasia, CD44v6 expression was strong and observed in $>75 \%$ of the columnar cells (Figure 4d). All dysplastic lesions displayed CD44v6 overexpression, characterized by strong immunoreactivity in $>75 \%$ of the cells. Findings were similar in low- and high-grade dysplasia, and the strong positivity in dysplasia changed abruptly to negativity in adjacent normal gastric epithelium (Figures 4e and f).

\section{v6-Containing CD44 Isoforms are Highly Expressed in Primary Sporadic Gastric Carcinomas}

The analysis of CD44v6 immunoexpression was performed in the 43 sporadic GCs. We found overexpression of v6containing CD44 isoforms in 27 of $43(62.8 \%)$ primary carcinomas (Table 1). Overexpression of v6-containing CD44 isoforms was not related with histotype of sporadic GCs: intestinal, 50\% (7/14); diffuse, 76.9\% (10/13); mixed, $62.5 \%$ $(10 / 16)$. CD44v6 was also observed in non-neoplasic tissue at the periphery of some GCs $(n=5$; two cases of hyperproliferative intestinal metaplasia at the periphery of intestinal carcinomas; three cases of foveolar hyperplasia at the periphery of two diffuse carcinomas and one mixed carcinoma).

In order to correlate the immunohistochemistry findings with the levels of mRNA expression of v6/v7-encoding CD44 transcripts, we performed real-time RT-PCR in 10 tumors, which displayed $>75 \%$ of CD44v6 $(+)$ cells, and matched non-neoplastic adjacent mucosa (Figures $5 \mathrm{a}$ and $\mathrm{b}$ ). We verified that all tumors showed higher mRNA expression levels of v6/v7-encoding CD44 transcripts in comparison with commercial RNA from normal stomach. Interestingly, in $40 \%$ of these primary tumor cases, the non-neoplastic adjacent mucosa displayed a statistically significant lower expression of $\mathrm{v} 6 / \mathrm{v} 7$-encoding CD44 transcripts than that found in tumors $(P<0.05)$ (Figure 5a).

\section{Diffuse GCs (Sporadic and Hereditary) Lacking E-Cadherin Expression Display High Expression of v6-Containing CD44 Isoforms}

E-cadherin, encoded by $\mathrm{CDH} 1$ gene, is the only molecule known to be structurally and epigenetically inactivated in a large proportion of sporadic and hereditary forms of diffuse GCs. ${ }^{21-31}$ The abovementioned observations, showing that $77 \%$ of the sporadic diffuse tumors displayed overexpression of v6-containing CD44 isoforms by immunohistochemistry, and that 4 of 10 cases showed a statistically significant increase of the v6/v7-encoding CD44 transcripts expression levels in the tumor (all of them of the diffuse type) compared with non-neoplastic adjacent mucosa, led us to explore a putative relationship between de novo expression of v6-containing CD44 isoforms and E-cadherin loss/aberrant expression.

E-cadherin was aberrantly expressed or lost in 58.1\% (25/43) of GCs: intestinal (42.9\%); diffuse $(76.9 \%)$; mixed (56.3\%). Overall, no statistically significant association was found between CD44v6 overexpression and E-cadherin loss/aberrant expression. In diffuse carcinomas, CD44v6 was overexpressed in tumors also displaying E-cadherin aberrant expression. In the only two cases of diffuse carcinoma that 

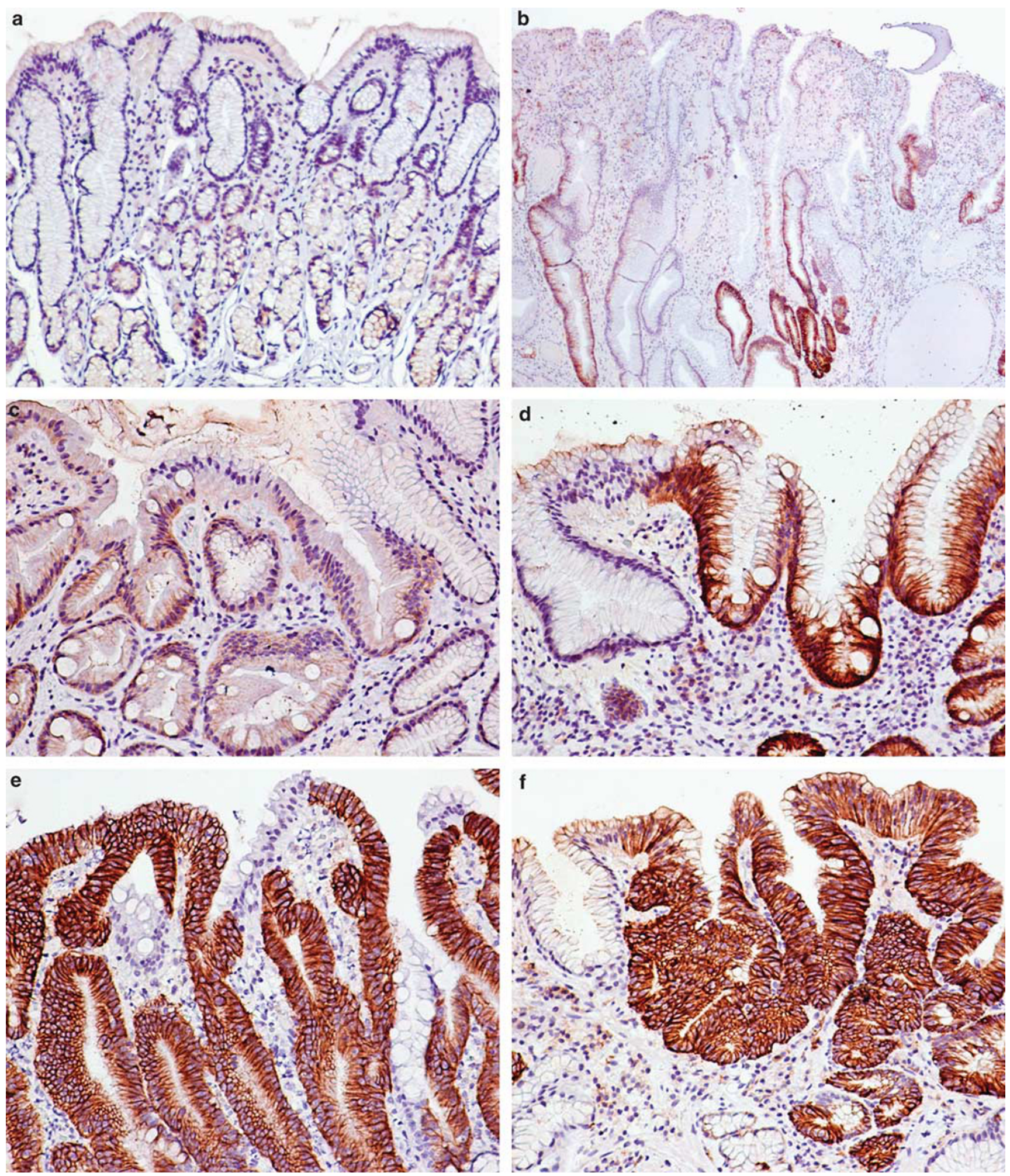

Figure 4 Immunohistochemical expression of CD44v6-protein isoforms in normal gastric mucosa, hyperplastic polyp, intestinal metaplasia and dysplasia. (a) Expression of CD44v6 in normal mucosa of the antrum displaying immunoreactivity in the cells of the neck zone of gastric glands ( $\times 100) ;(\mathbf{b})$ expression of CD44v6 in elongated/hyperplastic foveolae ( $\times 40)$; (c) expression of CD44v6 in $<25 \%$ of columnar cells in complete intestinal metaplasia ( $\times 200$ ); (d) overexpression of CD44v6 in >75\% of columnar cells in incomplete intestinal metaplasia ( $\times 200)$; (e) overexpression of CD44v6 in low-grade dysplasia: immunoreactivity is strong and displayed in $>75 \%$ of the cells; an abrupt change to lack of expression is observed at the adjacent normal epithelium ( $\times 200)$; (f) overexpression of CD44v6 in high-grade dysplasia $(\times 200)$ with features similar to those described in low-grade dysplasia (e). 
Table 1 Details on overexpression of CD44v6 and E-cadherin impairment in a series of $\mathbf{4 3}$ primary sporadic gastric carcinomas

\begin{tabular}{lccc}
\hline Histotype & $\begin{array}{c}\text { No. of } \\
\text { cases }\end{array}$ & $\begin{array}{c}\text { CD44v6 positive } \\
\text { expression (\%) }\end{array}$ & $\begin{array}{c}\text { E-cadherin aberrant } \\
\text { expression }\end{array}$ \\
\hline Diffuse & 13 & $10(76.9)$ & $10(76.9)$ \\
Mixed & 16 & $10(62.5)$ & $9(56.3)$ \\
Intestinal & 14 & $7(50.0)$ & $6(42.9)$ \\
Total & 43 & $27(62.8)$ & $25(58.1)$ \\
\hline
\end{tabular}

Criteria for CD44 expression: membranous expression in $>25 \%$ tumors cells $=$ positive. Criteria for E-cadherin expression: $>50 \%$ of cells with low membranous and/or cytoplasmatic expression $=$ aberrant. displayed complete absence of E-cadherin expression, there was also overexpression of v6-containing CD44 isoforms (Figure 6).

These observations led us to study diffuse gastric tumors from $\mathrm{CDH} 1$ germline mutation carriers that specifically developed HDGC due to complete loss of functional E-cadherin in their tumors. ${ }^{36}$ The expression of v6-containing CD44 isoforms was studied in three diffuse carcinomas from $\mathrm{CDH} 1$ germline mutation carriers. These three cases have been previously described to display $>75 \%$ cells with loss of E-cadherin expression, due to a combination of a germline mutation and methylation or $\mathrm{LOH}$ of the remaining wild-type allele. ${ }^{31}$ We verified that the three cases displayed overexpression of v6-containing CD44 isoforms expression,
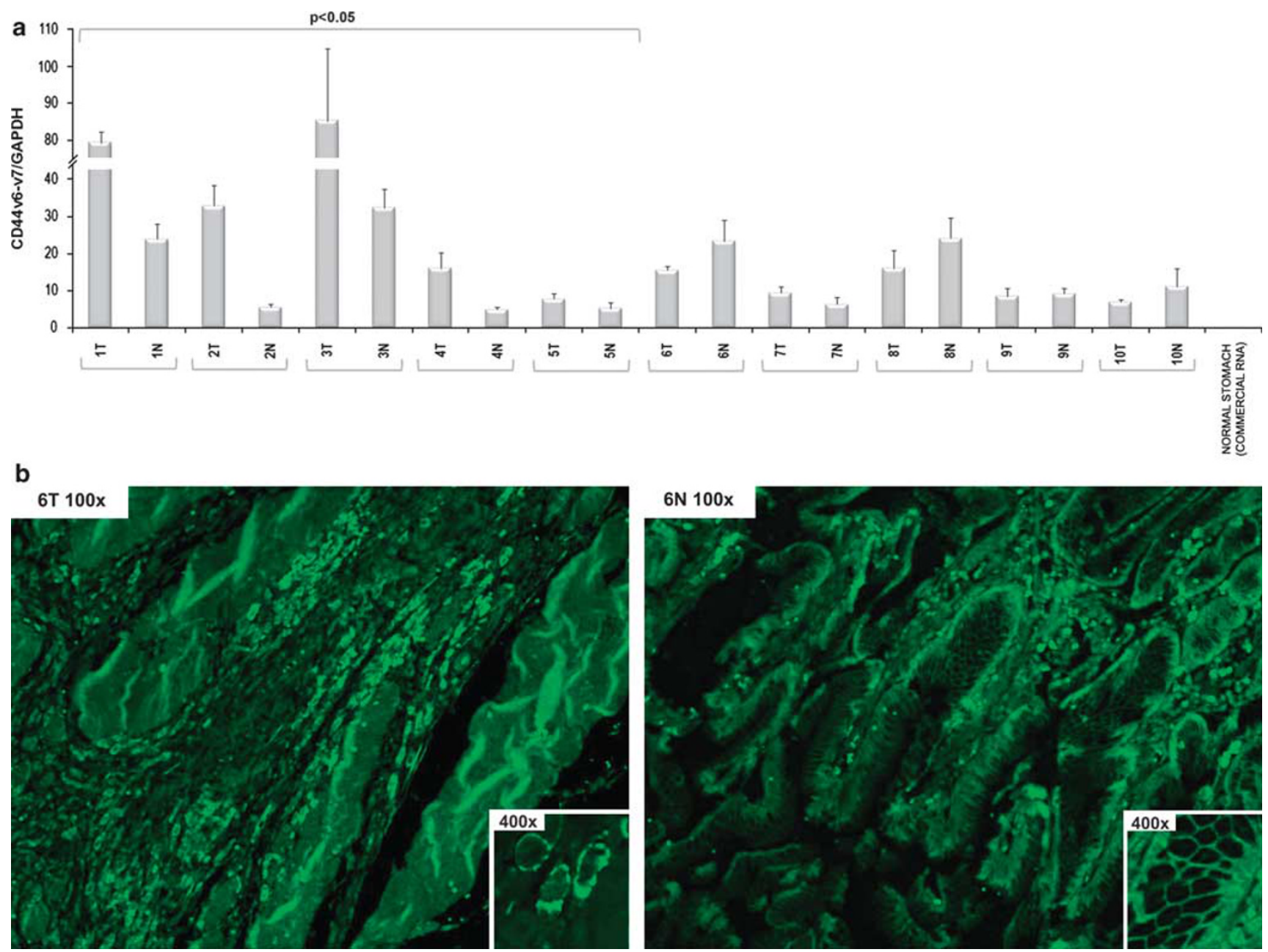

Figure 5 Expression quantification of v6/7-encoding CD44 transcripts and CD44v6 protein isoforms in sporadic primary GCs. (a) Quantitative target amplification of CD44v6/v7 mRNA transcripts performed using TaqMan gene expression assay. Normalization of the values of mRNA transcripts expression was made using human GAPDH endogenous control and s.d. values are displayed. RNA from normal stomach was used as control. Data are representative of three independent experiments. The ten sporadic gastric tumors were chosen based on positivity for CD44v6 immunoexpression (over 75\% expression). (b) Immunohistochemical expression of CD44v6-encoding isoforms observed in a sporadic diffuse/isolated cell GC (case number 6). Neoplasic cells show expression of CD44v6 variable isoforms. Both images were taken with $\times 100$ amplification. 


\section{a}

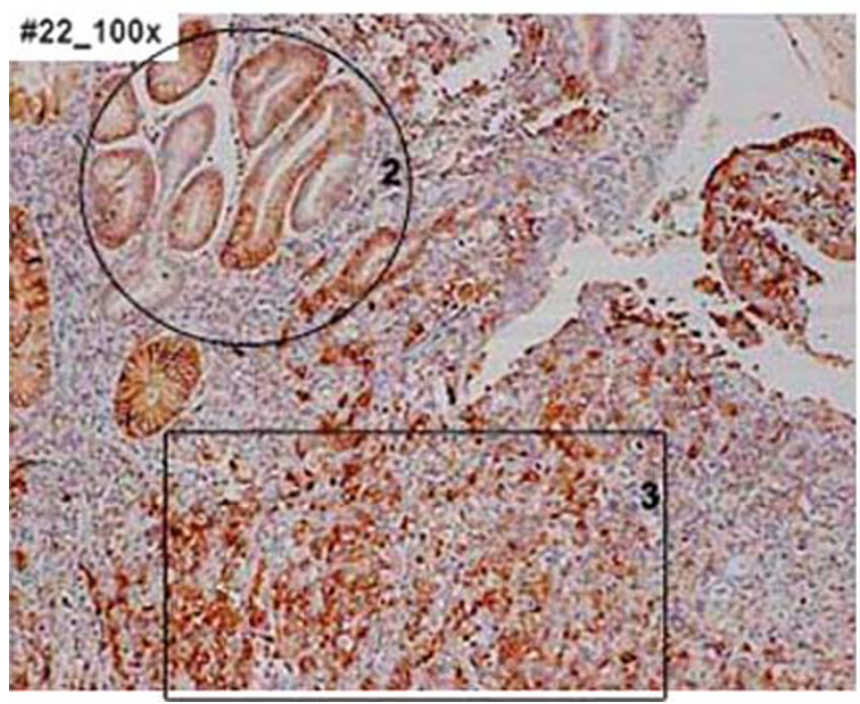

b

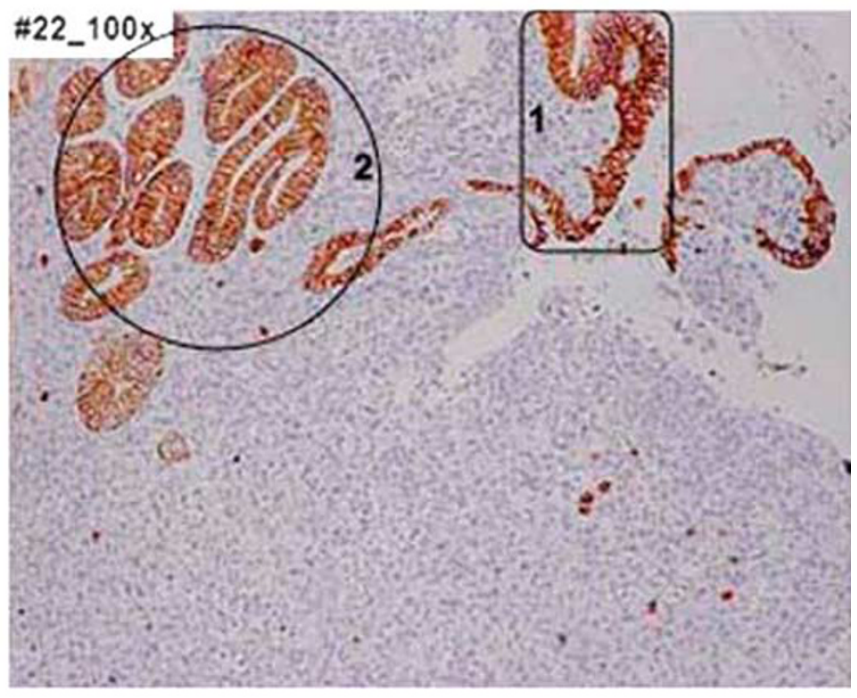

Figure 6 Immunohistochemistry analysis. (a) Expression of v6-protein CD44 isoforms; and (b) E-cadherin in one sporadic diffuse GC (case number 22). Normal glands show expression of E-cadherin only (rounded rectangle 1), hyperplastic glands show expression of both E-cadherin and CD44v6 (circles 2), and neoplastic cells show overexpression of CD44v6 variable isoforms and lack E-cadherin expression (rectangle 3). Images were taken with $\times 100$ amplification.
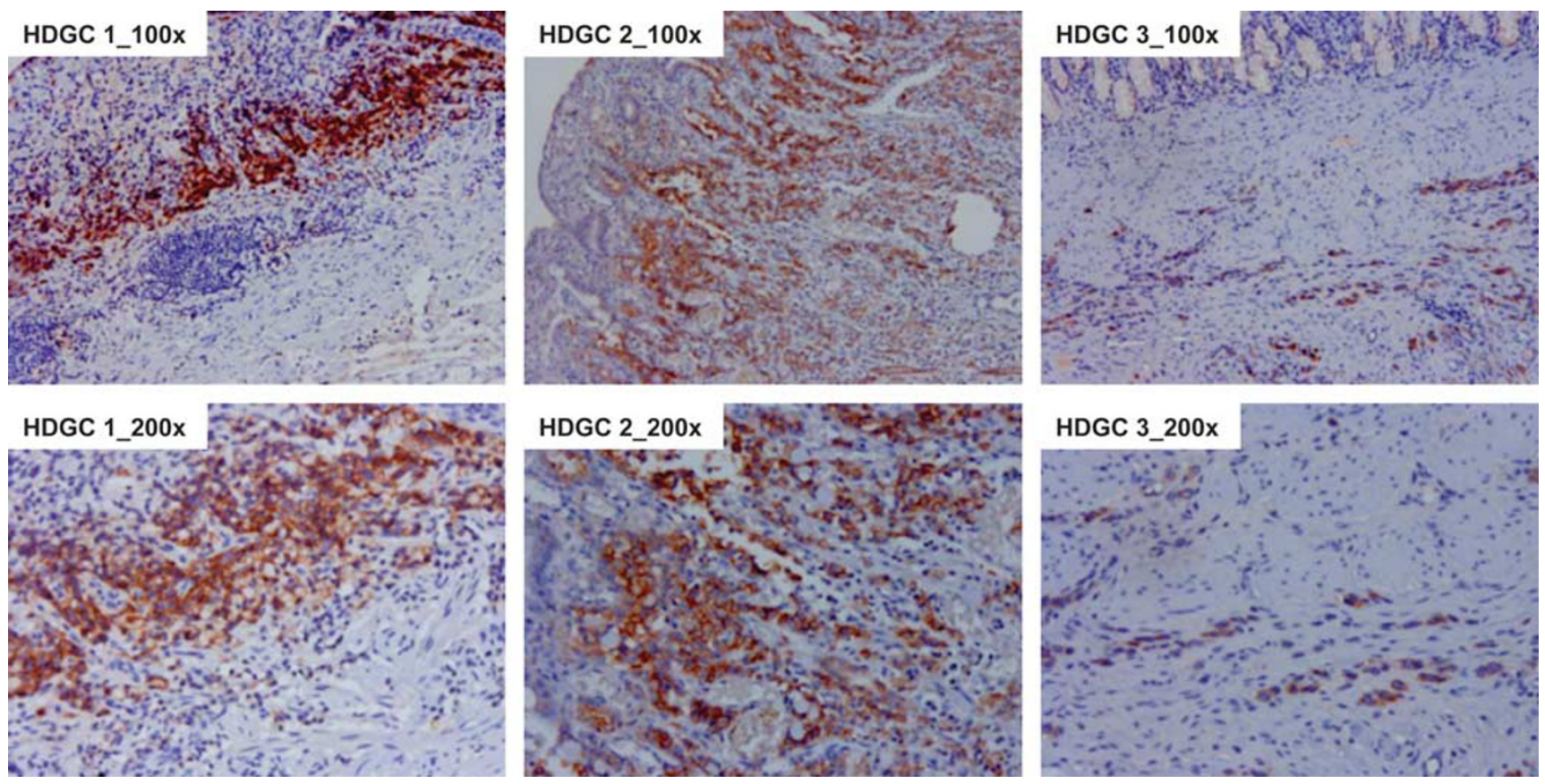

Figure 7 Immunohistochemical expression of CD44v6-protein isoforms in three HDGC tumors from CDH1 mutation carriers. Neoplasic cells show expression of CD44v6 variable isoforms. Upper row depicts images at $\times 100$ amplification and lower row depicts higher magnifications of the same image $(\times 200)$.

contrasting with the negativity in adjacent normal looking gastric mucosa (Figure 7).

\section{DISCUSSION}

In 1991, Günthert et $a l^{37}$ described for the first time the involvement of a CD44 variant in spontaneous metastatic behavior of nonmetastasizing rat tumor cell lines. CD44v6 has been shown to have a role as a major cellular matrix adhesion molecule, but it has also been demonstrated that CD44v6 leads to the activation of several signaling pathways associated with cancer progression. CD44v6 is required for HGF-induced c-Met activation and for the signal transfer 
from activated-MET to MEK and ERK. ${ }^{38}$ The mechanism of HGF-induced motility in primary human melanocytes has been demonstrated to be dependent on this c-Met-CD44v6 interaction, and HGF-mediated transcriptional upregulation of CD44v6 presumably mediated through a complex comprising NF- $\kappa \mathrm{B} / \mathrm{C} / \mathrm{EBP}-\beta$ and Egr- $1 .{ }^{39}$ Further, CD44v6 blocks Fas-mediated apoptosis; ${ }^{40}$ its constitutive expression provides a persistent growth stimulus ${ }^{41}$ or it interferes with PI3K/AKT pathways blocking apoptosis. ${ }^{42}$ As a consequence, the de novo expression of v6-encoding CD44 transcripts may bring a number of biological advantages to malignant gastric cells.

In the current report, we dissected the splicing modifications of the CD44 mRNA and its variant isoforms in GC cell lines and primary tumors (sporadic and hereditary), as well as in normal gastric mucosa and premalignant gastric lesions. We used real-time RT-PCR assays to quantitatively establish differences of mRNA transcripts expression, as well as fluorescent immunocytochemistry and immunohistochemistry to assess protein expression and localization.

The screening of the variable region of the CD44 in GC cell lines revealed a complex pattern of CD44 variable transcripts that was not found in normal stomach. Although only CD44s and $\mathrm{CD} 44 \mathrm{E}$ (standard and epithelial) forms were identified in normal stomach, larger CD44 transcripts encoding exon v6 were found in GC cell lines, with different expression levels. The comparison of v6/v7-encoding CD44 transcripts expression in GC cell lines and normal stomach showed that some GC cell lines expressed significant higher levels of these transcripts than normal stomach. Additionally, we confirmed that aberrant v6/v7-encoding CD44 transcripts expression gave rise to de novo expression of membranous CD44v6 protein in GC cell lines, but not in normal stomach from cancer-free individuals. In the latter, proliferative zones of non-neoplastic gastric glands (neck zones) showed CD44v6 positivity, as previously observed by others, in several epithelial tissues. ${ }^{43,44}$ In hyperplastic lesions (both sporadic hyperplastic polyps and hyperplastic lesions at the periphery of sporadic GCs), the expression of CD44v6 was observed in elongated foveolae and hyperproliferative intestinal metaplasia. Additionally, focal and faint expression was observed in deep glands of normal gastric mucosa and sporadic hyperplastic polyps. Therefore, CD44v6 variants are likely to have a normal and physiological role in the gastric epithelium, but an extension of this phenomenon into pathological contexts may occur in hyperproliferative lesions. Although overexpression of CD44v6 has been reported in intestinal metaplasia of gastric mucosa, ${ }^{45}$ to the best of our knowledge, this is the first time that overexpression of CD44v6 is described in hyperplastic lesions of the stomach. In other epithelial tissues such as the lung, ${ }^{46}$ endometrium $^{47,48}$ and breast, ${ }^{49}$ expression of CD44v6 in hyperplasia has already been observed. It remains to be clarified whether the overexpression of CD44v6 in hyperplastic/hyperproliferative lesions of gastric mucosa may indicate early premalignant changes not only in the abovementioned organs but also in the stomach. Supporting these observations, aged Atp4a(-/-) mice presenting chronic achlorhydria and hypergastrinemia develop progressive hyperplasia, mucocystic and incomplete intestinal metaplasia, with concomitant upregulation of CD44v6 before histological evidence of neoplasia. ${ }^{50}$

In intestinal metaplasia, CD44v6 overexpression was a feature of the incomplete type, which is considered to have a role in the cascade of events leading to GC of intestinal type. Further, in gastric dysplasia, which is an intraepithelial neoplasia, the expression of CD44v6 was strong and displayed $>75 \%$ of the cells. An abrupt loss of expression was observed in adjacent nondysplastic epithelium, supporting the view that dysplastic lesions are clonal. Altogether, these findings support the hypothesis that CD44v6 may be a marker useful to identify precursor lesions of GC.

The frequency of sporadic GC displaying overexpression of CD44v6 (62.8\%) was not different from that previously reported in other studies. ${ }^{51,52}$ At variance with other reports, in the present series, no significant relationship was found with histotypes of GC.

Our data on quantification of v6/7-encoding CD44 transcripts in a series of $10 \mathrm{CD} 44 \mathrm{v} 6$ expressing tumors clearly show that not only at the protein level, but also using specific RT-PCR, v6/7-encoding CD44 transcripts are significantly overexpressed in GCs in comparison with adjacent normal mucosa in at least $40 \%$ of the cases analyzed. Nevertheless, v6/7-encoding CD44 transcripts were similarly overexpressed in carcinomas and adjacent normal mucosa in $60 \%$ of the cases. As all the samples analyzed (10 tumor and 10 adjacent normal mucosa) displayed higher expression than the commercial RNA from normal stomach, we may speculate that CD44v6 overexpression occurs generally in GC and that nonneoplastic mucosa in the periphery of GC is frequently also under modification. Overall, our results from v6/7-encoding CD44 transcripts expression in 10 primary sporadic GC and in GC cell lines reproduced the results obtained with CD44v6 immunoexpression; therefore, we believe that the results from immunoexpression in material from the series of 43 primary sporadic GC demonstrate that CD44v6 is de novo expressed along the process of malignant transformation of gastric mucosa.

In the last years, a causal involvement of CD44 variants in metastases formation has been pursued. In two distinct studies, one using blocking antibodies against CD44v6 epitope ${ }^{53}$ and other using antisense mRNA targeting exon v6 of $\mathrm{CD} 44,{ }^{54}$ metastasis formation was blocked. A recent study aiming at characterizing which neoplastic cell population was generating peritoneal metastasis of GC demonstrated that GC stem cells were the metastasis precursors and that CD44 was one of the molecules that was significantly overexpressed in the cancer stem cell population constituting the bulk of peritoneal metastasis. ${ }^{55}$ These and other studies provided some evidences that CD44 might be involved in the 
invasiveness of tissues by malignant cells. One of the most important cancer invasion suppressor genes, E-cadherin gene $(C D H 1)$, is lost or impaired in the great majority of invasive sporadic and hereditary GCs; therefore, we tried to understand whether a correlation between E-cadherin impairment and CD44v6 expression could exist.

The expression of both proteins (CD44v6 and E-cadherin) was analyzed in 43 sporadic GC and three diffuse GC from HDGC patients, who are carriers of different $\mathrm{CDH} 1$ germline mutations. We observed that in E-cadherin-negative cases, CD44v6 was overexpressed. This result may be clinically relevant, mainly in the setting of $\mathrm{CDH} 1$ mutation carriers, as these patients are at increased risk of developing preclinic multifocal microscopic carcinomas that are virtually impossible to diagnose with currently used screening tools. ${ }^{56}$ In consequence, $\mathrm{CDH} 1$ mutation carriers are submitted to prophylactic gastrectomy while asymptomatic, ie, often before the third decade of life, with important consequences to their quality of life. Overall, carriers of $\mathrm{CDH1}$ germline mutations have a $>70 \%$ lifetime risk (penetrance) of developing diffuse GC. ${ }^{57}$ It is currently thought that very initial intramucosal cancer foci may remain indolent for undetermined periods of time; ${ }^{58}$ therefore, their early detection would greatly benefit $\mathrm{CDH} 1$ mutation carriers who elect to delay surgery, or for whom prophylactic gastrectomy (biopsy negative) is unacceptable, but gastrectomy with curative intent is acceptable when early foci of intramucosal carcinoma are diagnosed in gastric biopsies. CD44v6 can represent a target to allow detection of these foci at an early stage, if a CD44v6-specific probe is applied to currently available endoscopic and histological approaches to improve screening and diagnosis.

It remains to be known how a CD44v6-specific diagnosis can be designed as a noninvasive medical tool. For the last years nanomedicine has been emerging as a new powerful scientific field, bringing new answers for old unsolved challenges. Nanotechnology allows the development of nanodevices biomarker targeted for screening and early detection protocols, with the capability of avoiding biological and biophysical barriers. ${ }^{59}$ The development of a polymer-siRNA nanoparticle for silencing CD44v6 expression in GC cells in vitro has been reported. ${ }^{60}$ We herein hypothesize whether a new CD44v6-targeted approach can be explored to improve current endoscopic detection of premalignant and early malignant gastric lesions. Moreover, CD44v6 could be valuable to delimit areas of premalignant lesions before resection procedures. In the HDGC setting, a biomarker like CD44v6 could be useful to unreveal the presence of invasive malignant cells during routine histological screening of these patients.

In summary, in the present report we showed that CD44v6 overexpression in observed in the process of malignant transformation of gastric mucosa, from precursor lesions (incomplete intestinal metaplasia and adenomatous dysplasia) to advanced GCs. Moreover, we showed that CD44v6 overexpression also occurs in E-cadherin-negative tumors from HDGC $C D H 1$ germline mutation carriers, raising the hypothesis of using CD44v6 as a putative marker of early invasive intramucosal carcinoma in these patients.

\section{ACKNOWLEDGEMENTS}

We acknowledge Hugo Pinheiro for the help with the real-time RT-PCR assays. The work herein reported was funded by the Portuguese Foundation for Science and Technology (FCT) (Grant references PTDC/SAU-BEB/69901/2006 and PIC/IC/82923/2007; PhD Grant reference SFRH/BD/44982/2008 to C Cunha, and salary to C Oliveira in the frame of Program Ciência 2007).

\section{DISCLOSURE/CONFLICT OF INTEREST}

The authors declare no conflict of interest.

1. Aruffo A, Stamenkovic I, Melnick M, et al. CD44 is the principal cell surface receptor for hyaluronate. Cell 1990;61:1303-1313.

2. Screaton GR, Bell MV, Jackson DG, et al. Genomic structure of DNA encoding the lymphocyte homing receptor CD44 reveals at least 12 alternatively spliced exons. Proc Natl Acad Sci USA 1992;89: 12160-12164.

3. Ponta $H$, Wainwright $D$, Herrlich P. Molecules in focus The CD44 protein family. Int J Biochem Cell Biol 1998;30:299-305.

4. Rudzki Z, Jothy S. CD44 and the adhesion of neoplastic cells. Mol Pathol 1997;50:57-71.

5. Shtivelman E, Bishop JM. Expression of CD44 is repressed in neuroblastoma cells. Mol Cell Biol 1991;11:5446-5453.

6. De Marzo AM, Bradshaw C, Sauvageot J, et al. CD44 and CD44v6 downregulation in clinical prostatic carcinoma: relation to Gleason grade and cytoarchitecture. Prostate 1998;34:162-168.

7. Joensuu H, Klemi PJ, Toikkanen S, et al. Glycoprotein CD44 expression and its association with survival in breast cancer. Am J Pathol 1993;143:867-874.

8. Friedrichs $\mathrm{K}$, Kugler G, Franke F, et al. CD44 isoforms in prognosis of breast cancer. Lancet 1995;345:1237.

9. Kaufmann $\mathrm{M}$, Heider $\mathrm{KH}$, Sinn $\mathrm{HP}$, et al. CD44 variant exon epitopes in primary breast cancer and length of survival. Lancet 1995;345:615-619.

10. Mulder JW, Kruyt PM, Sewnath M, et al. Colorectal cancer prognosis and expression of exon-v6-containing CD44 proteins. Lancet 1994;344: 1470-1472.

11. Wielenga VJ, Heider $\mathrm{KH}$, Offerhaus GJ, et al. Expression of CD44 variant proteins in human colorectal cancer is related to tumor progression. Cancer Res 1993;53:4754-4756.

12. Terpe $H J$, Koopmann $R$, Imhof $B A$, et al. Expression of integrins and CD44 isoforms in non-Hodgkin's lymphomas: CD44 variant isoforms are preferentially expressed in high-grade malignant lymphomas. J Pathol 1994;174:89-100.

13. Takaishi S, Okumura $T$, Tu $S$, et al. Identification of gastric cancer stem cells using the cell surface marker CD44. Stem Cells 2009;27: 1006-1020.

14. Heider K-H, Dammrich J, Skroch-Angel P, et al. Differential EXpression of CD44 splice variants in intestinal- and diffuse-type human gastric carcinomas and normal gastric mucosa. Cancer Res 1993;53: 4197-4203.

15. Mayer B, Jauch KW, Gunthert U, et al. De-novo expression of CD44 and survival in gastric cancer. Lancet 1993;342:1019-1022.

16. Muller $\mathrm{W}$, Schneiders $\mathrm{A}$, Heider $\mathrm{KH}$, et al. Expression and prognostic value of the CD44 splicing variants v5 and v6 in gastric cancer. J Pathol 1997;183:222-227.

17. Mirecka J, Marx D, Schauer A. Immunohistochemical localization of CD44 variants 5 and 6 in human gastric mucosa and gastric cancer. Anticancer Res 1995;15:1459-1465.

18. Heider KH, Dammrich J, Skroch-Angel $\mathrm{P}$, et al. Differential expression of CD44 splice variants in intestinal- and diffuse-type human gastric carcinomas and normal gastric mucosa. Cancer Res 1993;53: 4197-4203.

19. Hong RL, Lee WJ, Shun CT, et al. Expression of CD44 and its clinical implication in diffuse-type and intestinal-type gastric adenocarcinomas. Oncology 1995;52:334-339. 
20. Ghaffarzadehgan K, Jafarzadeh M, Raziee HR, et al. Expression of cell adhesion molecule CD44 in gastric adenocarcinoma and its prognostic importance. World J Gastroenterol 2008;14:6376-6381.

21. Carneiro F, Huntsman DG, Smyrk TC, et al. Model of the early development of diffuse gastric cancer in E-cadherin mutation carriers and its implications for patient screening. J Pathol 2004;203: 681-687.

22. Suriano G, Mulholland D, de Wever O, et al. The intracellular E-cadherin germline mutation V832M lacks the ability to mediate cell-cell adhesion and to suppress invasion. Oncogene 2003;22:5716-5719.

23. Suriano $G$, Oliveira $C$, Ferreira $P$, et al. Identification of $C D H 1$ germline missense mutations associated with functional inactivation of the E-cadherin protein in young gastric cancer probands. Hum Mol Genet 2003;12:575-582.

24. Suriano G, Oliveira MJ, Huntsman D, et al. E-cadherin germline missense mutations and cell phenotype: evidence for the independence of cell invasion on the motile capabilities of the cells. Hum Mol Genet 2003;12:3007-3016.

25. Oliveira C, Bordin MC, Grehan N, et al. Screening E-cadherin in gastric cancer families reveals germline mutations only in hereditary diffuse gastric cancer kindred. Hum Mutat 2002;19:510-517.

26. Machado JC, Oliveira $C$, Carvalho $R$, et al. E-cadherin gene $(C D H 1)$ promoter methylation as the second hit in sporadic diffuse gastric carcinoma. Oncogene 2001;20:1525-1528.

27. Huntsman DG, Carneiro F, Lewis FR, et al. Early gastric cancer in young, asymptomatic carriers of germ-line E-cadherin mutations. N Engl J Med 2001;344:1904-1909.

28. Carneiro F, Machado JC, Seruca R, et al. E-cadherin changes in gastric carcimona. Histopathology 1999;35:477-478.

29. Mateus AR, Simoes-Correia J, Figueiredo J, et al. E-cadherin mutations and cell motility: a genotype-phenotype correlation. Exp Cell Res 2009;315:1393-1402.

30. Oliveira C, Senz J, Kaurah P, et al. Germline CDH1 deletions in hereditary diffuse gastric cancer families. Hum Mol Genet 2009;18:1545-1555.

31. Oliveira $\mathrm{C}$, Sousa S, Pinheiro $\mathrm{H}$, et al. Quantification of epigenetic and genetic second hits in $\mathrm{CDH} 1$ during hereditary diffuse gastric cancer syndrome progression. Gastroenterology 2009;136:2137-2148.

32. Lynch HT, Grady W, Suriano G, et al. Gastric cancer: new genetic developments. J Surg Oncol 2005;90:114-133; discussion 133.

33. Lauren P. The two histological main types of gastric carcinoma: diffuse and so-called intestinal-type carcinoma. An attempt at a histo-clinical classification. Acta Pathol Microbiol Scand 1965;64:31-49.

34. Filipe MI, Jass JR. Intestinal metaplasia subtypes and cancer risk. In: Gastric Carcinoma.Filipe MI, Jass JR (eds). Churchill Livingstone: London, 1986, pp 87-115.

35. Sato S, Yokozaki H, Yasui W, et al. Silencing of the CD44 gene by CpG methylation in a human gastric carcinoma cell line. Jpn J Cancer Res 1999;90:485-489.

36. Guilford P HJ, Harraway J, McLeod M, et al. E-cadherin germline mutations in familial gastric cancer. Nature 1998;26:6674. (referência incompleta!).

37. Günthert U, Hofmann M, Rudy W, et al. A new variant of glycoprotein CD44 confers metastatic potential to rat carcinoma cells. Cell 1991; 65:13-24.

38. Orian-Rousseau $\mathrm{Vr}$, Chen $\mathrm{L}$, Sleeman JP, et al. CD44 is required for two consecutive steps in HGF/c-Met signaling. Genes Dev 2002;16:3074-3086.

39. Damm SPK, Stefan M, Wels C, et al. HGF-Promoted Motility in Primary Melanocytes Depends on CD44v6 Regulated via NF-kappa B, Egr-1, and C/EBP-beta. J Investig Dermatol 2010;130:1893-1903.

40. Mielgo A, van Driel M, Bloem A, et al. A novel antiapoptotic mechanism based on interference of Fas signaling by CD44 variant isoforms. Cell Death Differ 2006;13:465-477.
41. Marhaba R, Bourouba M, Zoller M. CD44v6 promotes proliferation by persisting activation of MAP kinases. Cell Signal 2005;17:961-973.

42. Bates RC, Edwards NS, Burns GF, et al. A CD44 survival pathway triggers chemoresistance via lyn kinase and phosphoinositide 3-kinase/Akt in colon carcinoma cells. Cancer Res 2001;61:5275-5283.

43. Carvalho $R$, Milne AN, Polak $M$, et al. A novel region of amplification at 11p12-13 in gastric cancer, revealed by representational difference analysis, is associated with overexpression of CD44v6, especially in early-onset gastric carcinomas. Genes Chromosomes Cancer 2006;45: 967-975.

44. Heider $\mathrm{KH}$, Hofmann $\mathrm{M}$, Hors $\mathrm{E}$, et al. A human homologue of the rat metastasis-associated variant of CD44 is expressed in colorectal carcinomas and adenomatous polyps. J Cell Biol 1993;120:227-233.

45. Gulmann C, Grace A, Leader $M$, et al. CD44v6: a potential marker of malignant transformation in intestinal metaplasia of the stomach? An immunohistochemical study using tissue microarrays. Eur J Gastroenterol Hepatol 2003;15:981-986.

46. Wimmel A, Kogan E, Ramaswamy A, et al. Variant expression of CD44 in preneoplastic lesions of the lung. Cancer 2001;92:1231-1236.

47. Afify AM, Craig S, Paulino AF, et al. Expression of hyaluronic acid and its receptors, CD44s and CD44v6, in normal, hyperplastic, and neoplastic endometrium. Ann Diagn Pathol 2005;9:312-318.

48. Soon Cheol H, Jae Yun S, Jae Kwan L, et al. Significance of CD44v6 expression in gynecologic malignancies. J Obstet Gynaecol Res 2006;32:379-386.

49. Afify A, McNiel MA, Braggin J, et al. Expression of CD44s, CD44v6, and hyaluronan across the spectrum of normal-hyperplasia-carcinoma in breast. Appl Immunohistochem Mol Morphol 2008;16:121-127.

50. Judd LM, Andriga A, Rubio CA, et al. Gastric achlorhydria in $\mathrm{H} / \mathrm{K}$ ATPase-deficient (Atp4a(-/-)) mice causes severe hyperplasia, mucocystic metaplasia and upregulation of growth factors. J Gastroenterol Hepatol 2005;20:1266-1278.

51. Liu YJ, Yan PS, Li J, et al. Expression and significance of CD44 s, CD44v6, and nm23 mRNA in human cancer. World J Gastroenterol 2005;11: 6601-6606.

52. Polkowski WP, Skomra DG, Mielko J, et al. E-cadherin expression as predictive marker of proximal resection line involvement for advanced carcinoma of the gastric cardia. E J Surg Oncol 2004;30:1084-1092.

53. Seiter S AR, Reber S, Komitowski D, et al. Prevention of tumor metastasis formation by anti-variant CD44. J Experiment Med 1993; 177:443-455

54. Reeder JA, Gotley DC, Walsh MD, et al. Expression of ANTisense CD44 variant 6 inhibits colorectal tumor metastasis and tumor growth in a wound environment. Cancer Res 1998;58:3719-3726.

55. Nishii T, Yashiro M, Shinto O, et al. Cancer stem cell-like SP cells have a high adhesion ability to the peritoneum in gastric carcinoma. Cancer Sci 2009;100:1397-1402.

56. Carneiro F, Huntsman DG, Smyrk TC, et al. Model of the early development of diffuse gastric cancer in E-cadherin mutation carriers and its implications for patient screening. J Pathol 2004;203:681-687.

57. Pharoah PDP, Guilford P, Caldas C. The International Gastric Cancer Linkage $C$. Incidence of gastric cancer and breast cancer in $\mathrm{CDH} 1$ (E-cadherin) mutation carriers from hereditary diffuse gastric cancer families. Gastroenterology 2001;121:1348-1353.

58. Humar B, Fukuzawa R, Blair V, et al. Destabilized adhesion in the gastric proliferative zone and c-Src kinase activation mark the development of early diffuse gastric cancer. Cancer Res 2007;67:2480-2489.

59. Ferrari M. Cancer Nanotechnology: opportunities and challenges. Nat Rev Cancer 2005;5:161-171.

60. Wu YWW, Chen Y, Huang $\mathrm{K}$, et al. The investigation of polymer-siRNA nanoparticle for gene therapy of gastric cancer in vitro. Int J Nanomed 2010;5:129-136. 